\title{
Chefarzt klagt erfolgreich gegen Änderungskündigung Nicht jeder Chefarzt ist zwingend leitender Angestellter
}

Obwohl er in seinem Vertrag als „leitender Angestellter“ bezeichnet wird, konnte sich ein Chefarzt mit seiner Klage gegen eine Änderungskündigung durchsetzen, weil keine Zustimmung der Mitarbeitervertretung vorlag.

\section{Der Fall}

Der Kläger war seit Mai 2004 Chefarzt der Klinik für Innere Medizin. Die Beklagte ist Mitglied der Diakonie und betreibt ein evangelisches Krankenhaus. Für dieses Krankenhaus besteht eine Mitarbeitervertretung (MAV). Entsprechend seinem Anstellungsvertrag sollte der Kläger „im Sinne des Arbeitsrechts leitender Angestellter" sein. Die Beklagte hörte die MAV zu einer beabsichtigten ordentlichen Änderungskündigung an. Die MAV dankte daraufhin mit einem Antwortschreiben für die „umfassende Information“ und teilte mit, dass sie „für einen weiteren Austausch zur Verfügung“ stehe. Am Tag darauf kündigte die Beklagte das Arbeitsverhältnis des Klägers und bot ihm gleichzeitig die nahtlose Fortsetzung des Arbeitsverhältnisses als Chefarzt der medizinischen Klinik I - Allgemeine Innere, Diabetologie, Gastroenterologie, Hämato-/Onkologie - im selben Krankenhaus an.

Der Chefarzt nahm das Angebot unter Vorbehalt an und erhob fristgerecht Klage. Er begründete seine Klage damit, dass die Änderungskündigung unwirksam gewesen sei, weil die MAV ihr nicht zugestimmt habe und die $\mathrm{Zu}$ - stimmung auch nicht fingiert worden sei. Sowohl die erste also auch die zweite Instanz gaben der Klage des Chefarztes statt. Mit der Revision zum Bundesarbeitsgericht (BAG) verfolgt die Beklagte nach wie vor das Ziel, dass die Klage des Chefarztes abgewiesen wird und die Änderungskündigung wirksam war.

\section{Die Entscheidung des BAG}

Auch das BAG hält die Änderungskündigung für unwirksam, da die MAV nicht ordnungsgemäß beteiligt worden sei. Die Beklagte habe die Kündigung erklärt, bevor das durchzuführende Mitbestimmungsverfahren abgeschlossen gewesen sei.

\section{Chefarzt war kein leitender Mitarbeiter}

Der Senat ging davon aus, dass der Chefarzt kein leitender Mitarbeiter gewesen war, weshalb die ordentliche Kündigung des Arbeitnehmers nach Ablauf der Probezeit der eingeschränkten Mitbestimmung unterlag ( $\$ \S 41,42,44$ MVG-EKiR). Nach dem MVG-EKiR sind solche Personen - als zur Dienststellenleitung gehörend - von der Beteiligung der Mitarbeitervertretung in Personalangelegenhei-

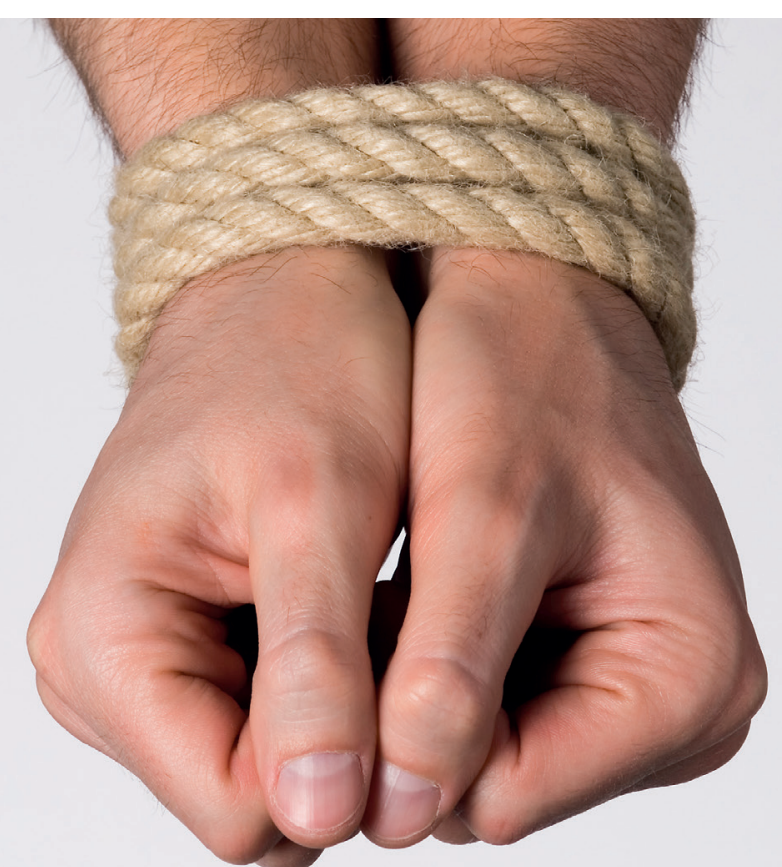

ten ausgenommen, die allein oder gemeinsam mit anderen ständig und nicht nur in Einzelfällen zu Entscheidungen in Angelegenheiten befugt sind, die nach dem MVG-EKiR der Mitberatung oder Mitbestimmung unterliegen. Entsprechend MVG-EKiR findet eine Beteiligung in Personalangelegenheiten nicht statt bei leitenden Mitarbeitern, die nach Dienststellung und Dienstvertrag im Wesentlichen eigenverantwortliche Aufgaben wahrnehmen, die ihnen regelmäßig wegen deren Bedeutung für den Bestand und die Entwicklung der Einrichtung im Hinblick auf besondere Erfahrungen und Kenntnisse übertragen werden.

Hieran fehlte es bei dem Chefarzt nach Auffassung des Senats. Allein die formale Stellung als Chefarzt und die Bezeichnung als „leitender Angestellter“ genügten nicht, um einen Beschäftigten als Mitarbeiter im Sinne des MVG-EKiR zu bezeichnen. Die Beklagte habe, um den Status des Klägers als leitenden Mitarbeiter zu begründen, lediglich auf die Regelungen des Anstellungsvertrages und auf die „gängige Praxis“ aller Einrichtungen im Geltungsbereich des Gesetzes, Chefärzte als leitende Mitarbeiter anzusehen, verwiesen. Weiteren Sachvortrag machte sie (verspätet) erstmals im Revisionsverfahren. Der Senat äußerte Zweifel daran, dass die Tätigkeit des Klägers von der Ausübung von Entscheidungsbefugnissen in beteiligungspflichtigen Angelegenheiten geprägt wurde. Auch folge aus seinem Anstellungsvertrag nicht, dass seine Tätigkeit schwerpunktmäßig durch eigenverantwortliche Wahrnehmung von für den Bestand oder die Entwicklung des Krankenhauses bedeutsamen Aufgaben bestimmt sein müsse. Dagegen spreche auch die Entwicklungsklausel in seinem Vertrag, die lediglich seine Anhörung vorsähe.

Folglich war eine eingeschränkte Mitbestimmung erforderlich.

\section{Fehler bei der Einbindung der MAV}

Nach Auffassung der Richter spricht 产 bereits Vieles dafür, dass die Beklagte $\because$ bereits das Beteiligungsverfahren nicht $\ddot{\overline{\bar{\omega}}}$ ordnungsgemäß eingeleitet hat. Denn sie 
hätte bei der MAV die Zustimmung zur beabsichtigten Kündigung beantragen müssen. Ihr Schreiben wurde jedoch lediglich als „Anhörung“ bezeichnet und es wurde um Stellungnahme gebeten. Da die MAV nach dem Vorbringen der Beklagten davon ausging, dass es sich um einen leitenden Mitarbeiter handelte, erschien es dem Gericht zweifelhaft, anzunehmen, dass die MAV davon ausging, es werde dennoch ihre Zustimmung zu der beabsichtigten Maßnahme erbeten. Fest stand für das Gericht, dass die Beklagte die Änderungskündigung erklärt hatte, bevor das Mitbestimmungsverfahren abgeschlossen war. Es lag weder eine Zustimmung der MAV vor, noch sei die Zustimmung ersetzt oder fingiert worden. So habe die MAV der beabsichtigten Kündigung weder ausdrücklich noch konkludent zugestimmt. In ihrem Schreiben sei keine Zustimmung zu sehen.

Auch eine Fiktion der Zustimmung war nicht anzunehmen. Zwar gibt es eine Vorschrift (38 Abs. 3 S.1 MVG-EKiR), wonach eine der eingeschränkten Mitbestimmung unterliegende Maßnahme als gebilligt gilt, wenn die MAV die erbetene Zustimmung nicht innerhalb von 2 Wochen schriftlich aus einem in $\S 41$ Abs. 2 MVG-EKiR aufgeführten Gründen verweigert oder eine mündliche Erörterung beantragt. Da die Kündigungserklärung 2 Tage nach dem Schreiben an die MAV ausgesprochen wurde, sei selbst eine auf das Mindestmaß von 3 Arbeitstagen abgekürzte Frist noch nicht abgelaufen gewesen.

Auch habe die Erklärung der MAV keinen vorzeitigen Eintritt der Fiktion bewirkt. Die Beklagte hätte die Kündigung selbst dann nicht vor Ablauf der Zweiwochenfrist aussprechen können, wenn die Erklärung der MAV als abschließende Äußerung anzusehen gewesen sein sollte. Eine Abkürzung der Äußerungsfrist und einen darauf bestehenden vorzeitigen Eintritt der Zustimmungsfiktion lasse das Gesetz nicht zu (aufgrund besonderer Normierung des MVG-EKiR).

Nicht entscheiden musste das BAG, ob die ordnungsgemäße Beteiligung der MAV ggf. überflüssig war. Nach Auffassung des Senats sind zwar Veränderungen des Klinikzuschnitts im Arbeitsvertrag der Parteien nicht ausgeschlossen. Deshalb käme es darauf an, ob mit der Zuweisung eines kleineren Zuständigkeitsbereichs die Grenzen des Direktionsrechts etwa dadurch überschritten würden, dass wegen erheblicher Einbußen bei den Liquidationserlösen der Kernbereich des Austauschverhältnisses betroffen wäre oder die neue Tätigkeit des Klägers sich gegenüber der Leitung der Klinik für - die gesamte - Innere Medizin nicht als gleichwertig darstellte (vgl. klinikarzt 2015; 44: 66-70).

Da aber bereits die Kündigungserklärung als solche an einem rechtlichen Mangel leide, könne einer Änderungsschutzklage trotz der Annahme des Änderungsangebots unter Vorbehalt auch dann stattgegeben werden, wenn die Änderungskündigung „überflüssig“ war.

\section{Keine Weiterbeschäftigung bei Annahme unter Vorbehalt}

Das Landesarbeitsgericht hatte dem Antrag des Chefarztes auf Weiterbeschäftigung stattgegeben. Soweit die Beklagte Revision hiergegen einlegte, gab das BAG ihr Recht. Ein Anspruch des Arbeitnehmers auf vorläufige Weiterbeschäftigung zu unveränderten Bedingungen scheide grundsätzlich aus, wenn er das Angebot auf Vertragsänderung unter Vorbehalt angenommen hat.

\section{Fazit}

Wer im Arbeitsrecht nicht die strengen Formalien beachtet, hat häufig das Nachsehen. Das Urteil macht deutlich, dass es nicht nur darauf ankommt, einen Arbeitnehmer als leitenden Angestellten zu bezeichnen, wenn er tatsächlich die Funktionen eines leitenden Angestellten gar nicht erfüllt. Deutlich wird darüber hinaus, dass es - nicht nur bei kirchlichen Häusern - äußerst wichtig ist, frühzeitig die notwendigen Argumente vorzubringen, da andernfalls - wie im hier vorliegenden Fall - der Ausschluss der Argumente droht. Bei erforderlicher Einbindung der MAV muss des Weiteren peinlich genau darauf geachtet werden, dass den Anforderungen auch tatsächlich Rechnung getragen wird.

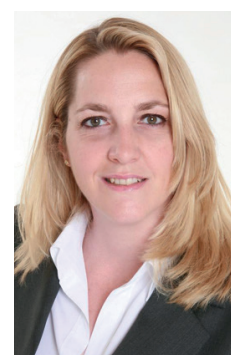

Korrespondenz Dr. iur. Isabel Häser Rechtsanwältin Fachanwältin für Medizinrecht Haimhauser Str. 1 80802 München E-Mail: haeser@kanzleihaeser.de 